\title{
Revolutionary Genome Editing Tools for Crop Improvement
}

\author{
Debanjana Saha ${ }^{1, *}$ Prajjal Dey ${ }^{2}$, Ankesh Sharma ${ }^{3}$ and Selukash Parida ${ }^{4}$ \\ ${ }^{1}$ Department of Biotechnology, Centurion University of technology and \\ Management Jatni, Bhubaneswar, Odisha-752020 India \\ ${ }^{2}$ Sri Sri University, Cuttack, Odisha-754006, India \\ ${ }^{3}$ Anand Agriculture University, Anand, Gujrat-388110, India \\ ${ }^{4}$ Odisha University of Agriculture and Technology, Bhubaneshwar, Odisha-751003, India \\ *Corresponding author
}

\section{A B S T R A C T}

\begin{tabular}{|l|}
\hline Ke y w o r d s \\
Agriculture, \\
Biotechnology, \\
Crop \\
\hline Article Info \\
\hline $\begin{array}{l}\text { Accepted: } \\
\text { 20 June } 2020 \\
\text { Available Online: } \\
\text { 10 July } 2020\end{array}$ \\
\hline
\end{tabular}

\section{Introduction}

In the present situation, the main problem faced by the human is to supply food security for the larger population of this world. In the year 2050, world production must be enhancing by 60-100 per cent to feed a population of 10 billion (FAOSTAT, 2016). Other than increased population, so many different factors like weather condition, less cultivable land availability, along with that biotic and abiotic stress also affects the farming condition and the production. Production of Staple crops like maize, rice, wheat and soybean can enhance by 38-67 per cent if the improvement of the condition prevail at the current rate(Ray et al., 2013). With the help of agronomic management practices like soil and water management, application of pesticides and fertilizer can improve the yield at a certain level. As arable land availability is less worldwide only agronomic practices alone cannot solve the problem. Development of new tools by the application of genetic engineering has strengthened the agronomic practices for breeding programmed to add improvement in production by some level. 


\section{Why genome editing is important?}

Hybridization is the foundation in any breeding programme for self-pollinating crops used by the plant breeders. Two parents with good attributes combine by hybridization to produce the superior quality of F1 than their parents; the process of this method is called Heterosis. This method is only applicable for closely related species or genus, and also unwanted traits are co-segregate with a trait of interest due to linkage, which are the shortcomings of this method. However, creating variation also can be done by the use of Mutation breeding. In mutation breeding without diminishing its agronomic and quality characteristics, the best cultivar variation can be created. To identify the function of genes also to understand the different biological pathway for crop improvement, physical, chemical and biological(T-DNA insertion or transposon) methods are can be use in the mutation breeding as a genetic exploitation tools(Ma et al., 2016). In mutation breeding sometimes whole part of the gene are not going to mutated which leads to create chimera, it takes lots of time to purify which is a major drawback of mutation breeding. Alternative to mutation breeding, transgenic also an important approach in plant breeding programmed. In this technique exact copy of gene of interest taken from any other source and incorporate into the host organism. But sometimes, integration of gene into the host organism can be non-specific, unstable and when it comes to eatable crop public concern comes into matter (Stephens and Barakate, 2017). Because of this benefits of genetic engineering technology confined to some cultivated crops.

For the last few years, as a modern technology genome editing tools are also using which is adventitious over genetic engineering. In genome editing technology, the main equipment is site-specific nucleases
(SSNs) that do the precise gene editing in host organism. SSNs mainly cause the doublestrand breaks (DSBs) in the specific DNA. To repair this double strand breaks mechanism use in this process are non-homologous end joining (NHEJ) or homology-directed recombination (HDR). Therefore it is known as genome editing. This leads to insertion/deletion (INDELS) and substitution mutations in the target area respectively (Jinek et al., 2012),

Use of Genome editing technology in plant breeding programme can create transgenefree varieties. As a genome-editing tool mainly two approaches are used like Zinc Finger Nuclease (ZFNs) and Transcription activator-like effector nucleases (TALENs) which are based on the fusion of DNA binding domain and nuclease for the editing of many crop genomes.

Result of transgenic can be random integration, sometimes random phenotyping results while in genome editing tools leads to generate specific mutants which make this tool an efficient approach in functional genomics and crop breeding programs. Crops generated through genome editing tool are more beneficial than transgenic crop as they contain their edited gene for the particular trait (Malzahn et al., 2017).

This genome editing technology is a collaboration of highly developed technology of molecular biology which assists in getting accurate, efficient, targeted improvement in the genomic region Genome editing technologies ZFNs and TALENs are used as a first-generation tools after that clustered regularly interspaced short palindromic repeats CRISPR-Cas9 technology came to spotlight as a second-generation genome editing tool, which provides simple and target specificity in gene editing. 
Genome alteration is done for few nucleotides in genome editing technology hence chance of risk involved in this process is less as compared to genetically modified crop. Also, the changes occurred in crops is a more likely natural population. Genome editing factors work as a tag unit. Once the factors are segregated out it is not possible to differentiate between spontaneous mutations and edit on a gene. Inbreeding programs these modified varieties can be used and these varieties will have more acceptability less regulatory issues and social concern than the genetically modified crops (Waltz, 2018).

Reflecting its broad importance, genome editing with engineered nucleases was named "Method of the Year" for 2011 by Nature Methods. So, the use of genome-edited tools is fast and precise in crop improvement thus provides a new direction to the advanced breeding program.

Here in this review, we will briefly discuss about various genome editing tools their mechanism and their application for crop enhancement.

\section{Zinc finger nuclease}

Zinc finger nuclease work as scissor to cut the DNA at a particular region and this principle worked in Gene editing technology. This zinc finger Nuclease editing tool is a combination of DNA recognition domain and a part of restriction enzyme FoK1 which the cleavage DNA at a particular location. This firstgeneration genome editing tools developed based on functional zinc finger domain where zinc present in between Cys2-His2. It gives stability to the structure as Zinc is a divalent positive ion which perfectly find with DNA. Every ZF bind in the DNA at three nucleotides and nuclease are forming groups to bind with the DNA at a specific sequence.

Basically every Cys2-His2 ZF domain contains 30 amino acids, which make structure in $\beta \beta \alpha$ pattern after folding (Petolino, 2015). Complete Zinc finger domain mainly composed of two different functional domains: $\mathrm{N}$ terminal region contain artificial zinc finger (Cys2-His2) and $\mathrm{C}$ terminal region have non-specific FoKI nuclease to cleave DNA domain. Dimerization FoKI nuclease is important in the function of Zinc Finger nuclease (Kim et al., 1996).

In order to make ZFNs more efficient to use as a genome editing tool, it is important to select modular designs for the application. The design of ZFNs, their assembly and optimization of $\mathrm{ZF}$ against a specific target DNA sequence, followed by combining single ZFs to target higher sequences. ZFNs have been effectively used in numerous organisms including plants (Gaj et al., 2013). ZFN used mainly for endogenous gene inactivation in Arabidopsis, high frequency modification of the Tobacco gene and highly used in maize for trait staking.

\section{Transcription activator-like effector nucleases}

In genome editing tools as an alternative, one more method has been introduced Known as transcription activator-like effector nucleases (TALENs). TALENs are occurring naturally from the plant pathogenic bacteria Xanthomonas which use to alter the gene expression in host plant cells. But TALENs also use as a tool in genome editing which contains artificial DNA binding domain and FoKI nuclease to create the cut in DNA at a particular region similar to ZFNs create double-strand breaks in the DNA.

TALEs have DNA binding domain which is highly conserved upto 30-35 amino acid except at the position of 12 and 13. The position of 12 and 13 which is not conserved is called repeat variable di-residue (RVD), 
which shows considerable correlation to recognize specific nucleotide. Like $\mathrm{ZFN}$, in TALENs also need to create Dimerization of FoKI nuclease to make the cut at specific area of DNA. Genome editing tool TALENs to work correctly there are some important requirements such as the presence of amino acid number between the DNA binding domain and the nuclease domain and also two separate the TALENs binding sites by the number of bases that may affect TALEN 's activity.

TALENs and ZFNs have comparable efficiencies when targeted to the same gene. Thus, the ease of design, high rates of cleavage activity, and the essentially limitless targeting range of TALENs make them suitable for the use by non-specialist researchers.

\section{Clustered regularly interspaced palindromic repeats}

A recently emerged modern genome editing method which has become extensively popular is the clustered regularly interspaced short palindromic repeats (CRISPR)/CRISPR associated (Cas) protein technique with the most notable being the CRISPR / Cas9 (based on Cas9 protein) but now CRISPR-Cpf1 is often used extensively. CRISPR / Cas works in prokaryotes as a type of adaptive immunity, developed over a long evolutionary period. This can destroy exogenous genes from an invading phage or plasmid and be first detected in Escherichia coli in 1987 identified a gap of around 32 nucleotides with non - overlapping sequences and "tandem repeats" downstream of the iap gene.In2002, the "tandem repeats" were called "clustered regularly interspaced short palindromic repeats" (CRISPR). In 2002, they called the "tandem repeats" "clustered regularly interspaced, short palindromic repeats"
(CRISPR). In2005, it was found that the CRISPR spacer sequence is highly homologous to exogenous sequences from bacterial plasmids and phages. CRISPR can cleave foreign DNA because of this homology between host and exogenous substances. The creation of the critical sitespecific gene-editing method called the CRISPR / Cas system. CRISPR / Cas needs only a short guide RNA strand to identify the appropriate loci by the Watson - Crick base pairing, the Cas endonuclease function may lead to genetic manipulation via removing the specific loci DNA And generating doublestranded DNA breaks (DSBs) which activate in vivo DNA repair mechanisms leading to gene mutation (e.g., insertion, deletion, and replacement).CRISPR / Cas is much more effective, and can simultaneously edit multiple target genes compared to previously evolved gene-editing methods like Zinc finger nucleases (ZFNs) and transcription activatorlike effector nucleases (TALENs).Depending on those advantages, CRISPR / Cas applications are growing rapidly. The geneediting tools ZFN and TALEN scan for appropriate protein sequences while CRISPR / Cas relies on guide RNA (gRNA).

CRISPR-Function of the immunity system is broken down into three levels. Injection of phage DNA into bacterial cells during CRISPR adaptation activates the Cas1-Cas2 adaptation module proteins which excise spacer-sized fragments of phage DNA and then channels for integration into CRISPR array. CRISPR array is transcribed during CRISPR RNA biogenesis, and the resulting pre-crRNA is processed to produced crRNAs at repeated sequences. The effectors of Cas proteins bound specific crRNAs. Once phage DNA with sequences matching a CRISPR spacer appears in the cell, effectors engineered by correct crRNA bind to that and cas executor nuclease destroys the resulting R-loop complex. 
Fig.1

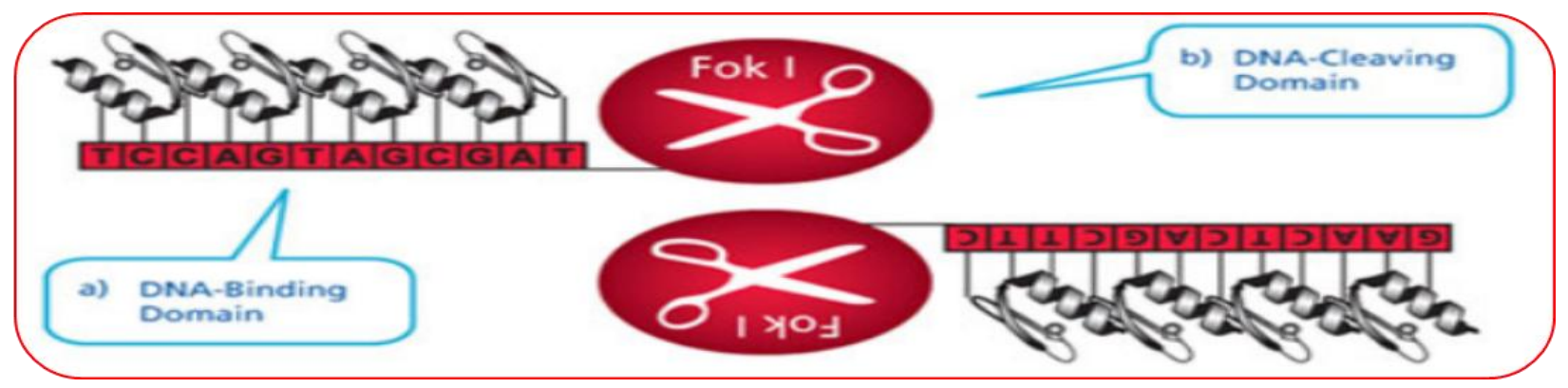

Fig.2

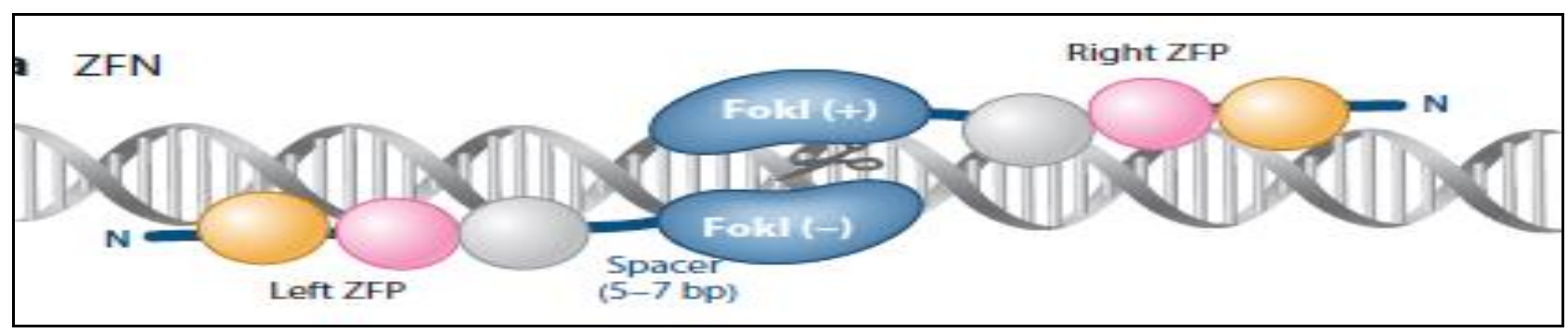

Illustration of zinc-finger nuclease (ZFN) pairs bound to DNA.

Fig.3

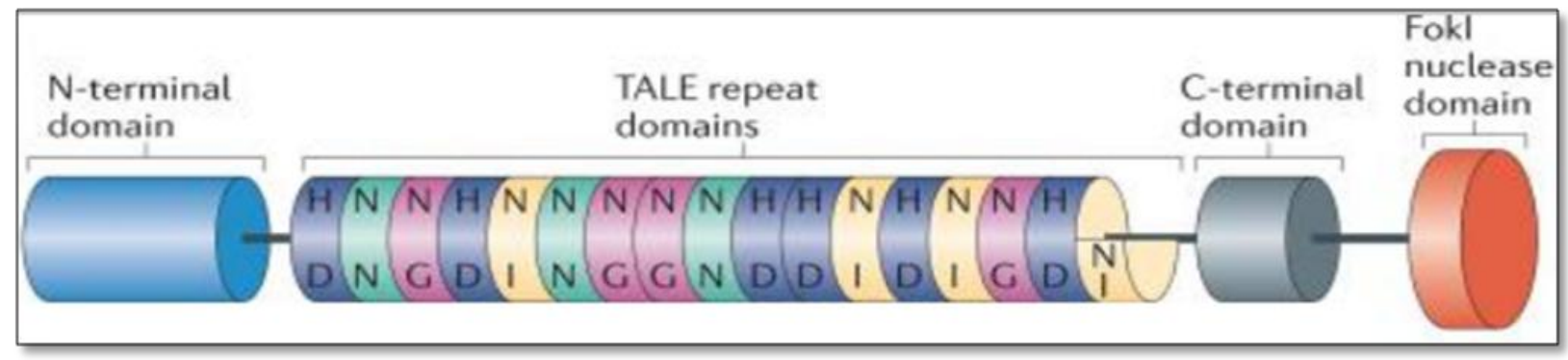

Schematic diagram of a TALEN.

Fig.4

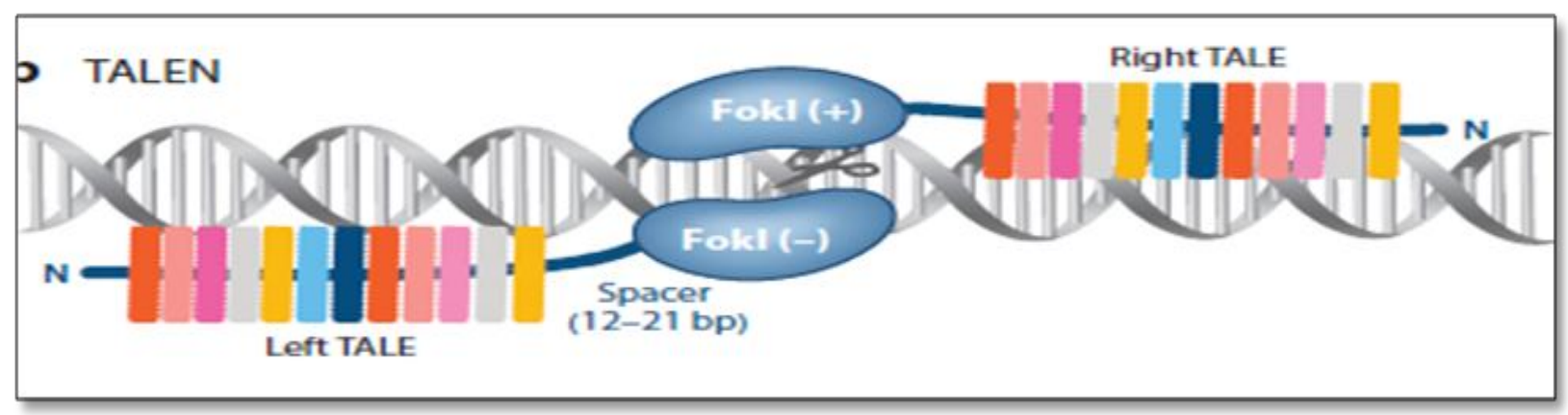

Representation of a transcription-activator-like effector nuclease (TALEN)pair bound to DNA. 
Fig.5

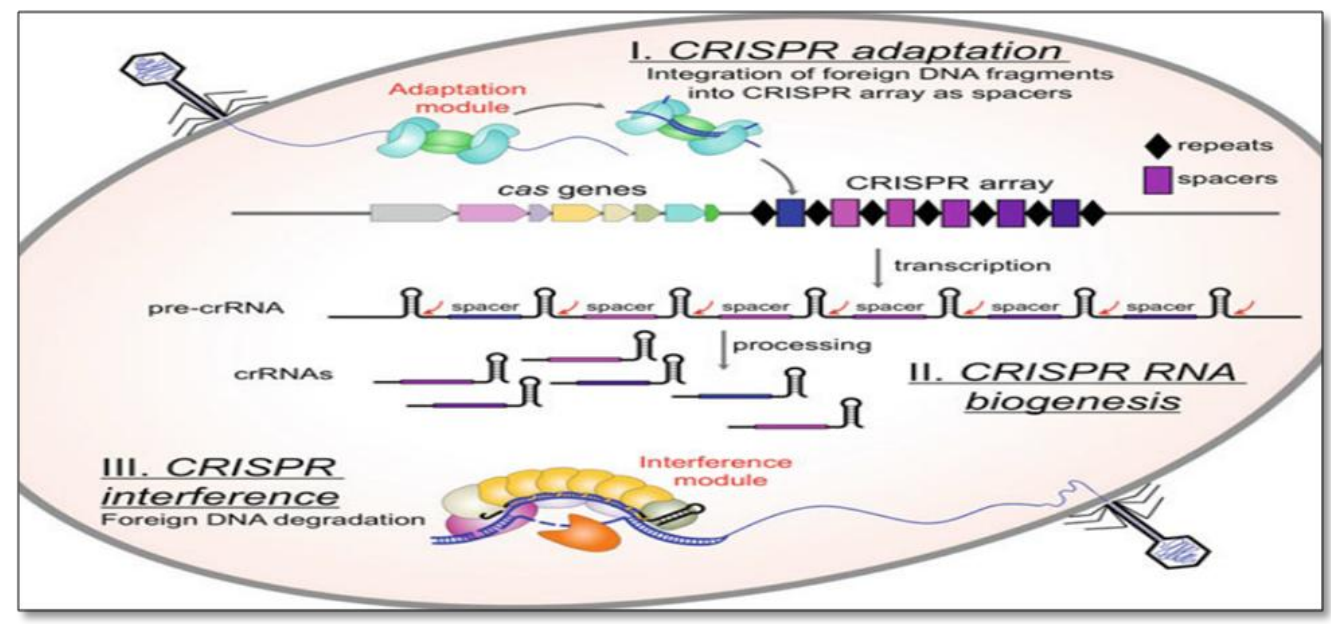

CRISPR-Cas adaptive immunity

Fig.6

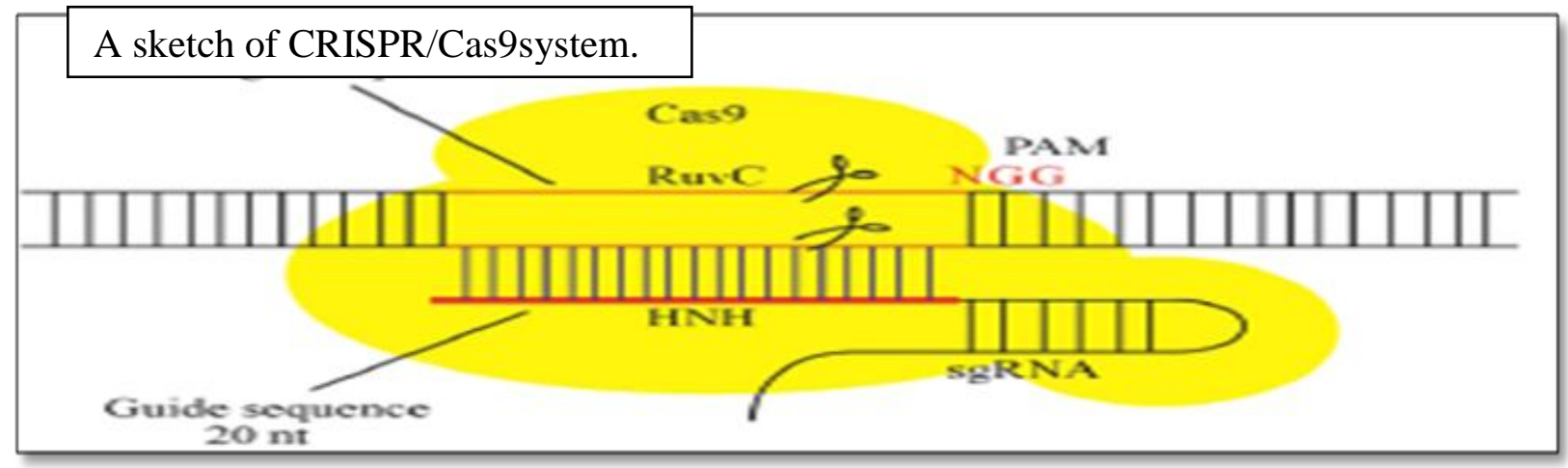

Fig.7

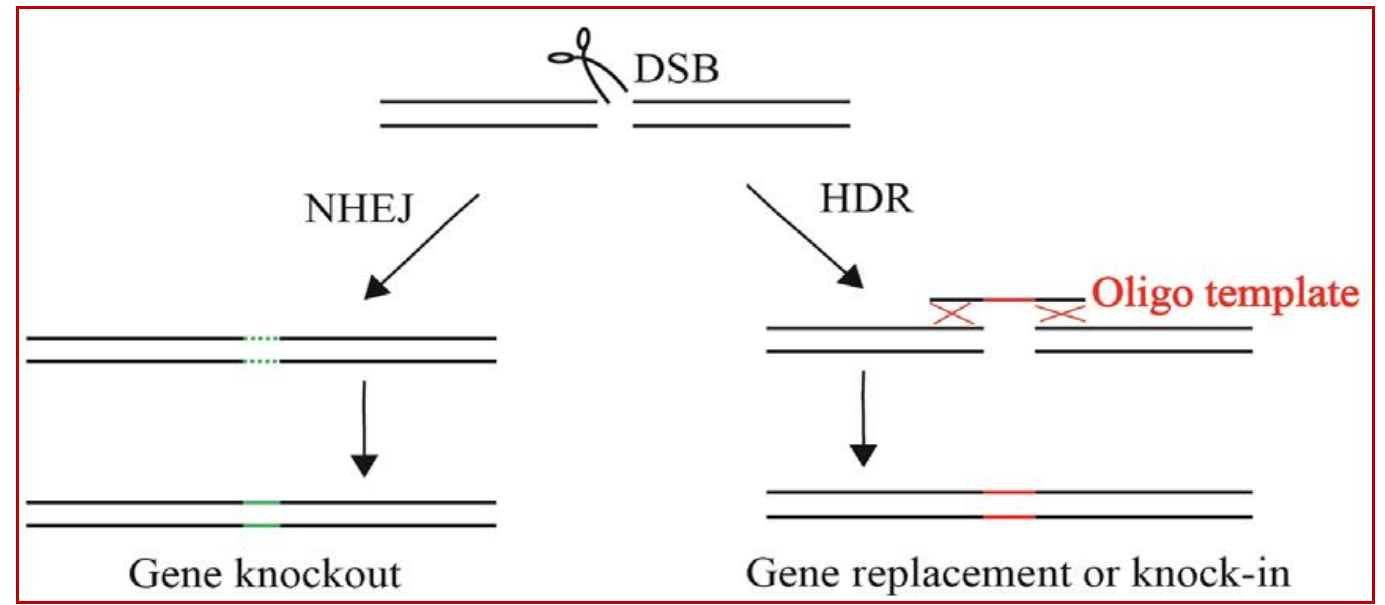


CRISPR / Cas can be broken down into three main types: I, II and III. Apparently, most work focuses more on the Type II CRISPR / Cas9 concepts and implementations than the other two types. The CRISPR / Cas9 method involves the editing of target genes by CASassociated 9 protein, crRNA (CRISPRRNA), tracrRNA (transactivatingcrRNA), and RNaseIII (RibonucleaseIII). Jinek et al., (2012),demonstrated that function of hybrid crRNA-tracrRNA are same as a single RNA (sgRNA) guide developed by fusion of crRNA to tracrRNA.

\section{The mechanism of CRISPR/Cas9}

CRISPR / Cas9 cut foreign DNA via two sections, Cas9 and sgRNA. Cas9 is a DNA endonuclease which can be derived from various bacteria, such as Brevibacillus laterosporus, Staphylococcus aureus, Streptococcus pyogenes, Streptococcus thermophilus and Streptococcus pyogenes. Cas9 includes two domains, namely the domain $\mathrm{HNH}$ and the domain RucV. The $\mathrm{HNH}$ domain splits the crRNA complementary strand, whereas the RucV as domain cleaves the double-stranded DNA opposite strand. The sgRNA is an approximately100nt long, synthetic RNA. Its 5 / end has a 20nt sequence which serves as a reference sequence to classify the target sequence followed by an adjacent protospacer motif (PAM) sequence, which is also the NGG consensus (N, anynucleotide; G, guanine).The loop structure at the sgRNA's 30 -end can attach the target gene through the guide sequence and establish a Cas9 complex, which cleaves the double-stranded DNA and forms a double-strand break(DSB) at this site

Nearly 5000 articles were published between 2010 and 2018, detailing the use of CRISPR. SgRNA design has become one of the critical factors in the successful editing of target genes using CRISPR / Cas9 to the maximum.
Implementation of a CRISPR project entails simple steps, viz. I the discovery of the PAM sequence in the target gene, (ii) the synthesis of a single gRNA (sgRNA), (iii) the cloning of sgRNA into a capable binary vector, (iv) the incorporation of transformation into host species / cell lines accompanied by (v) the screening and (vi) the verification of edited lines. The easy steps associated in CRISPR / Cas9 mediated genome editing (CMGE) permit only a small laboratory with such a fundamental transformation of plants set up to carry out projects for genome editing. In the last half decade, CRISPR / Cas9 techniques were used more extensively to edit plant genomes compared with ZFNs / TALENs.

\section{Cleaved genomic sites repair}

Repairing nuclease-created DNA breaks is an important step in the process of genome editing. Endogenous cellular mechanisms repair DNA breakage: non-homologous endjoining (NHEJ) or homology-dependent (or directed) repair (HDR) (Hsu et al., 2013). Through NHEJ, which is the simplest mechanism, the ends of the cleaved DNA are joined together, often resulting in the insertion or deletion of nucleotides (indels), while further fluctuating the gene reading frame, resulting in a knockout gene (Perkin et al., , 2016).However, HDR is a tool in which a sequence comprising homology to target is used as a template to repair the break or DNA lesion. Thus, by giving a model consisting of the desired sequence of concern lined by sequences homologous both to sides of the breaking point, one may force that preferred sequence to be inserted into the target location. Once HDR arises, homologous recombination is necessary to allow for innovative gene recovery or insertion sequences (Perkin et al., 2016).

In conclusion to meet the demand for food, feed, fibre, medicine and biofuels plants are 
always used as a source. Conventional breeding has been used for several years to enhance the properties of domestic crops. Diverse approaches, such as traditional breeding, genetic manipulation or genome editing, help to develop plants by altering different alleles. However, genome editing targets a particular location in the genome, so it requires less screening or backcrossing. Enhancement of the food and feed production, medicines, animal models, genetic variation, materials, power, gene surgery can easily be achieved by genome modification tool genome editing.

\section{References}

FAOSTAT (2016). FAOSTAT Database. Available at:

http://faostat3.fao.org/faostatgateway/go/to/download/Q/QC/E.

Gaj, T., Gersbach, C.A., Barbas III, CF, 2013. ZFN, TALEN, and CRISPR/Cas-based methods for genome engineering. Trends Biotechnol. 31 (7), 397-405. T. Shah et al. Plant Physiology and Biochemistry 131 (2018) 12-21 20

Hsu, P.D., Scott, D.A., Weinstein, J.A., et al., 2013. DNA targeting specificity of RNA guided Cas9 nucleases. Nat. Biotechnol. 31, 827-832.

Jinek M, Chylinski K, Fonfara I, Hauer M, Doudna JA, Charpentier E. A programmable dual-RNA-guided DNA endonuclease in adaptive bacterial immunity. Science. 2012;337:816-21.

Kim, Y.G., Cha, J., Chandrasegaran, S., 1996.
Hybrid restriction enzymes: zinc finger fusions to Fock I cleavage domain. Proc. Natl. Acad. Sci. USA 93 (3), 1156-1160.

Ma X., Zhu Q., Chen Y., Liu Y. G. (2016). CRISPR/Cas9 platforms for genome editing in plants: developments and applications. Mol. Plant 9 961-974.

Malzahn A., Lowder L., Qi Y. (2017). Plant genome editing with TALEN and CRISPR. Cell Biosci. 7:21.

Pavletich, N.P., Pabo, C.O., 1991. Zinc finger-DNA recognition: crystal structure of a Zif268-DNA complex at 2.1A. Sci. Technol. Humanit. 252 (5007), 809-817.

Perkin, L.C., Adrianos, S.L., Oppert, B., 2016. Gene disruption technologies have the potential to transform stored product insect pest control. Insects 7 (3) 46.

Petolino, J.F., 2015. Genome editing in plants via designed zinc finger nucleases. Vitro Cell Dev. Biol. Plant 51 (1).

Stephens J., Barakate A. (2017). "Gene editing technologies - ZFNs, TALENs, and CRISPR/Cas9," in Encyclopedia of Applied Plant Sciences 2 Edn eds Thomas B., Murray B. G., Murphyp D. J. (Cambridge, MA: Academic Press; ) 157-161.

Waltz E. (2018). With a free pass, CRISPRedited plants reach market in record time. Nat. Biotechnol. 36 6-7.

Zhang, F., Wen, Y., Guo, X., 2014. CRISPR/Cas9 for genome editing: progress, implications and challenges. Hum. Mol. Genet. 23 (1), R40-R46.

\section{How to cite this article:}

Debanjana Saha, Prajjal Dey, Ankesh Sharma and Selukash Parida. 2020. Revolutionary Genome Editing Tools for Crop Improvement. Int.J.Curr.Microbiol.App.Sci. 9(07): 2162-2169. doi: https://doi.org/10.20546/ijcmas.2020.907.252 